J. Hano

Nagoya Math. J.

Vol. 72 (1978), 65-69

\title{
ON A THEOREM OF LICHNEROWICZ
}

\author{
JUN-ICHI HANO*
}

In his study on the structure of the complex Lie algebra of holomorphic vector fields on a compact Kähler manifold, Lichnerowicz ([3] Theorem 2, see also [1] and [4]) shows that if the first Chern class of the manifold is positive semi-definite, then to each harmonic (0.1)-form (i.e. anti-holomorphic 1-form) $\eta$, there exists a holomorphic vector field $X$ such that the (0.1)-form $\iota(X) k$ is $d^{\prime \prime}$-cohomologous to $\eta$, where $k$ is the Kähler form. The purpose of this note is to indicate that this result is a consequence of an existence theorem for solutions of a certain selfadjoint elliptic partial differential equation.

1. Let $M$ be a connected compact Kähler manifold of complex dimension $n$. Let

$$
g=\sum g_{\alpha \beta} d z^{\alpha} d \bar{z}^{\beta}
$$

and

$$
k=\sum i g_{\alpha \beta} d z^{\alpha} \Lambda d \bar{z}^{\beta}
$$

be the fundamental tensor field and the Kähler form respectively. Let $\mathfrak{a}$ be the complex Lie algebra of holomorphic vector fields on $M$, and $\mathfrak{i}$ the ideal of $a$ consisting of holomorphic vector fields $X$ such that for any holomorphic 1-form $\omega, \omega(X)=0$.

Take $X \in \mathfrak{a}$, then $\iota(X) k$ is a form of bidegree $(0.1)$ and $d^{\prime \prime}$-closed. By a theorem of Hodge

$$
\iota(X) k=H_{\iota}(X) k+d^{\prime \prime} \lambda,
$$

where $H_{\iota}(X) k$ is the harmonic component of $\iota(X) k$ and $\lambda$ is a function on $M$. It is known that $X \in \mathfrak{i}$ if and only if $H_{\iota}(X) k=0$ (Lichnerowicz [3]). Thus, we have an injective linear map of $\mathfrak{a} / \mathfrak{i}$ into the complex

Received June 22, 1977.

* Partially supported by N.S.F. Grant \#MPS 72-04880A3. 
vector space $\mathfrak{h}^{0,1}$ of anti-holomorphic 1 -forms. The dimension of $\mathfrak{h}^{0,1}$ is the one half of the first Betti number $b_{1}(M)$.

THEOREM (Lichnerowicz [3]). If the first Chern class of a connected compact Kähler manifold $M$ is positive semi-definite, then there is a subalgebra $\mathfrak{b}$ of $\mathfrak{a}$ of dimension equal to $\frac{1}{2} b_{1}(M)$ such that

$$
\mathfrak{a}=\mathfrak{i}+\mathfrak{b}, \quad \mathfrak{i} \cap \mathfrak{b}=0
$$

and that each vector in $\mathfrak{b}$ is nowhere vanishing.

2. Let us take an arbitrary volume element $v$ on $M$, a positively oriented nowhere vanishing $(n, n)$-form. In terms of a local holomorphic coordinates $\left(z^{\alpha}\right)$,

$$
v=(i)^{n^{2}} K d z^{1} \wedge \cdots d z^{n} \wedge d \bar{z}^{1} \wedge \cdots \wedge d \bar{z}^{n} .
$$

Define a real closed (1.1)-form $\alpha_{v}$ on $M$ by

$$
\alpha_{v}=\frac{i}{2 \pi} \sum \frac{\partial^{2} \log K}{\partial z^{\alpha} \partial \bar{z}^{\beta}} d z^{\alpha} \wedge d \bar{z}^{\beta}
$$

Then, the cohomology class $\left[-\alpha_{v}\right]$ is the first Chern class of the manifold $M$.

Given a vector field $X$, let us denote by $\delta_{v}(X)$, the divergence of $X$, namely, $\theta(X) v=\delta_{v}(X) v$. The formula

$$
\delta_{v}(f X)=f \delta_{v}(X)+X f
$$

for a function $f$ is useful. From the above definition of $\alpha_{v}$, it follows easily that if $X$ is a holomorphic vector field and $Y$ a vector field of bidegree (1.0),

$$
\alpha_{v}(X, \bar{Y})=-\bar{Y} \delta_{v}(X)
$$

(Koszul [2]). Utilizing the Kähler connection $\nabla$ on $M$ and the property of the divergence above, we obtain the following formula for $\alpha_{v}$ valid for any vector field $X$ of bidegree (1.0),

$$
2 \pi i \alpha_{v}(X, \bar{X})=-\bar{X} \delta_{v}(X)+\delta_{v}\left(\nabla_{X} X\right)-\rho(X),
$$

where, in terms of a holomorphic local coordinates $\left(z^{\alpha}\right)$,

$$
\rho(X)=\sum_{\alpha, \beta} \frac{\partial \bar{\xi}^{\beta}}{\partial z^{\alpha}} \cdot \frac{\partial \xi^{\alpha}}{\partial \bar{z}^{\beta}}, \quad \text { for } X=\sum \xi^{\alpha} \frac{\partial}{\partial z^{\alpha}} .
$$


For later use, we examine $\rho(X)$ further and claim that if $d^{\prime \prime} \iota(X) k$ $=0$, then $\rho(X) \geqq 0$. Indeed, if this is the case, we see easily that

$$
\rho(X)=g\left(\nabla^{\prime \prime} X, \overline{\nabla^{\prime \prime} X}\right) \geqq 0 \text {. }
$$

By Stokes' Theorem, $\int_{M} \theta(\bar{X})\left(\delta_{v}(X) v\right)=0$ and hence from (1) it follows that

$$
\int_{M} 2 \pi i \alpha_{v}(X, \bar{X}) v=\int_{M}\left|\delta_{v}(X)\right|^{2} v-\int_{M} \rho(X) v .
$$

3. Now let us assume that the first Chern class of $M$ is positive semi-definite. This means that we can choose a volume element $v$ so that $2 \pi i \alpha_{v}(X, \bar{X}) \geqq 0$ for any vector field $X$ of bidegree (1.0). First, we prove that the map of $\mathfrak{a} / \mathfrak{i}$ into $\mathfrak{h}^{0.1}$ in $\mathbf{1}$ is onto, in other words, given an anti-holomorphic 1-form $\eta$, there is a holomorphic vector field $X$ such that

$$
H_{\iota}(X) k=\eta .
$$

For this purpose, we show that given an anti-holomorphic 1-form $\eta$, we can choose a function $\lambda$ on $M$ so that a vector field $X$ of bidegree (1.0) determined by

$$
\iota(X) k=\eta+d^{\prime \prime} \lambda
$$

is of zero divergence, and hence is holomorphic on account of (2) and (3).

From (4),

$$
\theta(X) k=d d^{\prime \prime \lambda}
$$

and

$$
\theta(X) k^{n}=\left(\frac{1}{2} \Delta \lambda\right) k^{n},
$$

where $\Delta$ denotes the Laplacian associated to the Kähler metric $g$. Put $v=e^{f} k^{n}$ with a real valued function $f$ on $M$.

$$
\delta_{v}(X) v=\theta(X) v=\left(X f+\frac{i}{2} \Delta \lambda\right) v .
$$

Define vector fields $Y$ and $Z$ of bidegree (1.0) by 


$$
\iota(Y) k=\eta \text { and } \iota(Z) k=d^{\prime \prime} \lambda
$$

Then $X=Y+Z, Z=-\sum i g^{\alpha \bar{\beta}} \frac{\partial \lambda}{\partial \bar{Z}^{\beta}} \frac{\partial}{\partial Z^{\alpha}}$ and

$$
X f=Y f-i g\left(d^{\prime \prime} \lambda, d^{\prime} f\right) \text {. }
$$

Therefore, for the vector field $X$ defined by $(4), \delta_{v}(X)=0$ if and only if $\lambda$ satisfies the equation

$$
\Delta \lambda-2 g\left(d^{\prime \prime} \lambda, d^{\prime} f\right)=-Y f
$$

where $f$ and $Y f$ are given. Moreover

$$
\int_{M}(-Y f) v=0
$$

In order to see the above equality, we remark that $\eta$ is harmonic. Thus

$$
\theta(Y) k=d_{\iota}(Y) k+\iota(Y) d k=d \eta=0
$$

and

$$
0=\int_{M} \theta(Y) v=\int_{M}(Y f) v+\int_{M} e^{f} \theta(Y) k^{n}=\int_{M}(Y f) v
$$

Put

$$
D \lambda=\Delta \lambda-2 g\left(d^{\prime \prime} \lambda, d^{\prime} f\right) .
$$

Then, $D$ is an elliptic differential operator of degree 2. By a straight forward computation, we see that $D$ is self-adjoint with respect to the inner product

$$
\langle\lambda, \mu\rangle=\int_{M} \lambda \bar{\mu} v
$$

The condition (6) means that the function $-Y f$ is orthogonal to the eigen space of $D$ belonging to the eigen value 0 , which consists of constant functions on $M$. Therefore, the equation (5) has a unique smooth solution up to an additive constant ([5] Theorem, p. 43).

We have seen that for each $\eta \in \mathfrak{h}^{0,1}$, there is a unique holomorphic vector field $X$ such that $H_{\iota}(X) k=\eta$ and that $\delta_{v}(X)=0$.

4. Let $\mathfrak{b}$ be the set of all holomorphic vector fields $X$ on $M$ whose 
divergence $\delta_{v}(X)=0$. Let us show that $\mathfrak{b} \cap \mathfrak{i}=(0)$, which is valid under no assumption on the first Chern class of $M$.

If $X \in \mathfrak{i}$, then $\iota(X) k=d^{\prime \prime} \lambda$ and $k(X, \bar{X})=\bar{X} \lambda$. By Stokes' theorem, $\int_{M} \theta(\bar{X})(\lambda v)=0$. Hence

$$
\int_{M} k(X \bar{X}) v=-\int_{M} \lambda \delta_{v}(\bar{X}) v=0 .
$$

If $X \in \mathfrak{b} \cap \mathfrak{i}$, then from (7) it follows that $X=0$.

In our case where the first Chern class is positive semi-definite, $\operatorname{dim} \mathfrak{b}=\frac{1}{2} b_{1}(M)$. We have finished the proof of the theorem.

Remark. Another theorem of Lichnerowicz ([2] Theorem 1) asserts that if the first Chern class of $M$ is negative semi-definite, namely if $2 \pi i \alpha_{v}(X, \bar{X}) \leq 0$ for a certain volume element $v$, then $\mathfrak{i}=(0)$. This fact follows immediately from (3) and (7), both being valid without any assumption on the first Chern class. Indeed, if $X \in \mathfrak{i}, \delta_{v}(X)=0$ on account of (3) and hence $X=0$ by (7).

\section{REFERENCES}

[1] S. Kobayashi, Transformation groups in differential geometry, Berlin-HeidelbergNew York, Springer 1972.

[ 2 ] J.-L. Koszul, Sur la forme hermitienne canonique des especes homogènes complexes, Canad. J. Math. 7 (1955), 562-576.

[ 3 ] A. Lichnerowicz, Variétés kahleriennes et première classe de Chern, J. Diff. Geom. 1 (1967), 195-224.

[4] Y. Matsushima, Holomorphic vector fields on compact Kähler manifolds, Conf. Board Math. Sci. Regional Conf. Ser. in Math. 7 (1971), A. M. S.

[ 5 ] P. Gilkey, The index theorem and the heat equation, 4 Berkeley, Publish or Perish 1974.

Washington University 